\title{
Standing tall-risk of oesophageal adenocarcinoma decreases with height
}

A new study has demonstrated, perhaps contrary to expectations, that height is inversely associated with the risk of oesophageal adenocarcinoma and Barrett oesophagus. Increased height is associated with an increased risk of some other cancers, so Aaron Thrift and colleagues decided to investigate the relationship between height and oesophageal cancer and its precursor, Barrett oesophagus.

"Our primary interest was to determine whether taller stature is associated with increased risk of oesophageal adenocarcinoma, and if so, if it explained the striking male predominance of oesophageal adenocarcinoma (7:1 male:female ratio)," explains Thrift, who is corresponding author of the study published in Clinical Gastroenterology and Hepatology.

The researchers analysed epidemiological and genetic data from 999 patients with oesophageal adenocarcinoma, 2,061 patients with
Barrett oesophagus and 2,168 population controls. They used Mendelian randomization methods to obtain unconfounded and unbiased estimates of the association with height.

The team found that increased height was actually associated with a reduced risk of oesophageal cancer and Barrett oesophagus, and that this association was not due to confounding from known risk factors or bias.

"Further research is needed to determine how shorter stature is associated with increased risk of oesophageal adenocarcinoma," concludes Thrift. "For now, we are planning to incorporate height into existing risk models for these diseases."

Isobel Leake

Original article Thrift, A. P. et al. Risk of esophageal adenocarcinoma decreases with height based on consortium analysis and confirmed by Mendelian randomization. Clin. Gastroenterol. Hepatol. doi:10.1016/j.cgh.2014.01.039 